\title{
Identification Using Classification Analysis of Flunitrazepam in Necrophagous Larvae via Differential Pulse Voltammetry and Fluorescence Excitation-Emission Matrix (EEM) Spectroscopy
}

\author{
Leomir A. S. de Lima, ${ }^{a}$ Camilo L. M. Morais, ${ }^{b}$ Jéssica T. Jales, ${ }^{c}$ Renata A. Gama, ${ }^{c}$ \\ Sherlan G. Lemos ${ }^{d}$ and Kássio M. G. Lima*,a \\ ${ }^{a}$ Química Biológica e Quimiometria, Instituto de Química, \\ Universidade Federal do Rio Grande do Norte, 59078-970 Natal-RN, Brazil \\ ${ }^{b}$ School of Pharmacy and Biomedial Sciences, Lancashire University, \\ Preston PRI 2HE, United Kingdom \\ ${ }^{c}$ Departamento de Microbiologia e Parasitologia, \\ Universidade Federal do Rio Grande do Norte, 59072-970 Natal-RN, Brazil \\ ${ }^{d}$ Departamento de Química, Universidade Federal da Paraíba, \\ 58051-390 João Pessoa-PB, Brazil
}

\begin{abstract}
The use of insects to identify drugs in a cadaver has often been explored in the field of entomotoxicology. There are accurate methods; however, they require a high cost and are very time-consuming. The objective of this study was to develop two methods based on differential pulse voltammetry (DPV) and fluorescence excitation-emission matrix (EEM) spectroscopy to classify necrophagous larvae (Chrysomya megacephala, C. albiceps, Lucilia sp. and Cochliomyia macellaria) containing flunitrazepam. The voltammograms of larval extract samples were analyzed by principal component analysis (PCA), successive projection algorithm (SPA) and genetic algorithm (GA): linear and quadratic discriminant analysis (LDA and QDA). The EEM fluorescence data from larval extract samples were analyzed by $2 \mathrm{D}$ and parallel factor analysis (PARAFAC) with LDA. These results suggest that DPV and EEM combined with chemometrics can be used as tools for the classification of flunitrazepam in fly larvae presenting innovative applications.
\end{abstract}

Keywords: differential pulse voltammetry, fluorescence spectroscopy, flunitrazepam, entomotoxicology, necrophagous larvae

\section{Introduction}

Forensic entomology is the science that investigates traces of insects and other arthropods for criminal, legal or civil investigative purposes. ${ }^{1}$ It is often requested for estimation of minimum postmortem interval. ${ }^{2,3}$ However, recent techniques have allowed the collection of robust entomological evidence to resolve questions about the corpse movement, submersion interval, decapitation or dismemberment time, identification of specific traumas and artifacts postmortem cells. ${ }^{4,5}$ More recently, the identification of drug of abuse or other chemicals important to the crime scene was achieved in insects, which gave rise to a new research field, Entomotoxicology. ${ }^{6,7}$ Flunitrazepam

*e-mail: kassiolima@gmail.com has been reported as the rape date drug. Its illicit consumption added to alcoholic beverages strengthens its sedative effects, favoring the action of criminals, in which the victims become defenseless due to the loss of muscular control and loss of consciousness, being vulnerable to assaults and rape. Illicit use of flunitrazepam is also common in nightclubs, in which youngsters and adults use the drug individually or in combination with other drugs such as cocaine and heroin.

In skeletons or bodies in an advanced stage of decomposition, biological materials such as blood, urine or internal organs may not be available. Considering the necrobiontophagous nature of some Diptera larvae, when feeding of tissue of the corpses of intoxicated humans, they introduce in their own metabolism toxins or drugs used by the individuals when in life. Thus, by receiving target substances 
from the human organism, some insects, especially fly larvae, can serve as an alternative for toxicological analysis. ${ }^{8}$

In addition to the identification of drugs and toxins in corpses and the development of immature insects, entomotoxicology may be useful in investigating the effects of such substances on the development of arthropods in order to assist in a more precise postmortem estimation, since the presence of certain chemical substances may influence the decay process of a cadaver. The increase in the number of drug-related deaths, especially by abuse of heroin and cocaine or deaths connected to accidental or suicidal consumption of poisons or toxic substances, justifies the substantial interest in forensic entomology, especially entomotoxicology. ${ }^{9}$

Insects and other arthropods can be analyzed by standard toxicological procedures, such as radioimmunoassay, gas chromatography, thin-layer chromatography, high performance liquid chromatography (HPLC), and gas chromatography-mass spectrometry. ${ }^{1,8,10}$ However, forensic toxicology analysis requires simpler and faster methods for the screening of chemical substances that aid in the elucidation of crimes, ${ }^{10}$ for example, using attenuated total reflectance Fourier transform infrared (ATR-FTIR) spectroscopy ${ }^{11}$ or near infrared spectroscopy. ${ }^{12}$

Electrochemical techniques are powerful and versatile analytical tools that offer high sensitivity, precision, with a large linear dynamic range and low-cost instrumentation. The development of more sensitive electrochemical techniques allows its application in the detection of chemical substances such as drugs, both in its crude form and added to biological samples. ${ }^{13}$ Voltammetric techniques are examples of electrochemical methodologies used in the identification and dosage of pharmaceutical compounds in various forms (tablets, capsules, injections, suspensions) as well as in biological samples. ${ }^{13-23}$

Voltammetric measurements are simple and easily performed. ${ }^{13}$ In this sense, the present report proposes a simple, low cost and high precision electrochemical method to detect the presence of flunitrazepam in necrophagous larvae using differential pulse voltammetry (DPV). Voltammograms were submitted to multivariate classification analysis, linear discriminant analysis (LDA) and quadratic discriminant analysis (QDA), aided by data dimensionality reduction algorithms: principal component analysis (PCA), successive projections algorithm (SPA) and genetic algorithm (GA). The proposed combination of voltammetry and chemometrics was evaluated in the identification of necrophagous larvae containing flunitrazepam.

Several groups have applied fluorescence spectroscopy to identify spectral characteristics that correspond to the presence of target analytes in complex mixtures. The main focus of these approaches is to identify drug abuse such as flunitrazepam in fly larvae that have fed on a decomposing victim of a crime, the so-called "rape date", where it is possible to compare specimens with and without flunitrazepam through an analysis of their spectral profiles. In this context, the application of multivariate analysis is considered a powerful tool to improve classification or quantification analysis. For example, fluorescence excitationemission matrix (EEM) has been used in oil samples to monitor the content of polycyclic aromatic compounds, ${ }^{24}$ developing a method to facilitate discrimination of diesel fuel with rebate tax from oil that is illegally processed by the sorption process. ${ }^{25}$ It has also been used the EEM fluorescence combined with parallel factorial analysis (PARAFAC) to develop a quantitative method for analyzing simulated amphetamine-type illegal drugs. ${ }^{26}$ Satisfactory results were found for the simultaneous determination of methamphetamine and 3,4-ethylenedioxymethamphetamine in the presence of the drug.

This paper reports a method using DPV and fluorescence spectroscopy combined with multivariate analysis to discriminate control and flunitrazepam contamination in larvae of necrophagous flies. EEM data classification was performed using unfolded $\mathrm{LDA},{ }^{27} \mathrm{QDA}^{27}$ and support vector machines (SVM). ${ }^{28}$ These methodologies were compared with three-way EEM data using 2D algorithms, including parallel factor analysis with linear discriminant analysis (PARAFAC-LDA) ${ }^{29}$ and two-dimensional linear discriminant analysis (2D-LDA). ${ }^{30}$

\section{Experimental}

\section{Animals used in the experiment}

The experiments were performed with 32 Wistar rats (Rattus norvegicus) having an average weight of $255 \pm 30 \mathrm{~g}$ (Ethics Committee Approval Protocol 044/2013). The rats with age of $60 \pm 5$ days used at the beginning of the experiment from the vivarium of the Department of Biophysics and Pharmacology of the Federal University of Rio Grande do Norte were accommodate (4 animals per cage) in nursery cages with free access to water (or ethanol solutions) and food (Purina ${ }^{\circledR}$, Labina). The rats were divided into four groups with 8 rats each, where each group received different treatment: $(i)$ ethanol, in which ethyl alcohol (Alcoolabor ${ }^{\circledR}$, Segmenta) was used in increasing concentrations of $2 \%$ ( 3 days), $4 \%$ (3 days), $6 \%$ (15 days) totaling a period of 21 days $;^{31}$ (ii) flunitrazepam, dosage of $2 \mathrm{mg} \mathrm{kg}^{-1}$ orally; ${ }^{32}$ (iii) conjugated (ethanol plus flunitrazepam), ethyl alcohol (Alcoolabor $®$, Segmenta) was used in increasing concentrations of $2 \%$ (3 days), 
$4 \%$ ( 3 days), $6 \%$ (15 days) totaling a period of 21 days $^{31}$ and received doses of $2 \mathrm{mg} \mathrm{kg}^{-1}$ flunitrazepam orally; ${ }^{32}$ (iv) control, received only water ad libitum. After one hour of administration of flunitrazepam, the rats were euthanized by guillotine decapitation and each rat was placed in individualized plastic bags. Animals belonging to the four treatments were transported separately.

In the field, the smell traps containing the rats were distributed on an 8 grid (grid distance of approximately $50 \mathrm{~m}$ and at $1.50 \mathrm{~m}$ high from the ground) along one of the trails in the $7^{\text {th }}$ Combat Engineering Battalion (The Brazilian Army), an Atlantic forest area located in the city of Natal, Brazil. Each grid contained one rat of each treatment (control, ethanol, flunitrazepam, and conjugate) distant $5 \mathrm{~m}$ apart. Traps remained in the field for five consecutive days. On the third and fourth days, 10 immatures were collected (resulting from oviposition, prioritizing the largest larvae, representing the first generations that arrived in the carcass) of each trap. The larvae were placed in a glass vial containing glycerin and sent to the laboratory for identification and chemical analysis.

A set of 3 larvae was added to $2 \mathrm{~mL}$ of dimethyl sulfoxide (DMSO), then triturated, homogenized and centrifuged. The supernatant solution was stored in a cryotube and then sent for analysis. Two groups were formed for classification, (i) control plus ethanol, larvae collected in carcasses of the control and ethanol groups; (ii) flunitrazepam, larvae collected on flunitrazepam and conjugate groups. In total, 40 samples (20 control and 20 containing flunitrazepam) were directed to DPV and fluorescence EEM analysis.

The analyzes were performed with larvae of Chrysomya megacephala, C. albiceps, Lucilia sp. and Cochliomyia macellaria from the $3^{\text {rd }}$ instar, with specimens of each species in both control and flunitrazepam groups.

\section{Electrochemical analysis}

\section{Differential pulse voltammograms}

All DPV measurements were performed by a portable bipotentiostat/galvanostat $\mu$ Stat 400 (DropSens, Spain), controlled by DropView software, and carried out in $0.1 \mathrm{~mol} \mathrm{~L}^{-1}$ phosphate buffer at $\mathrm{pH}=7$, from +1.0 to $-1.0 \mathrm{~V}$, scan rate of $15 \mathrm{mV} \mathrm{s}^{-1}$, pulse time of $50 \mathrm{~ms}$ and pulse amplitude of $100 \mathrm{mV}$. The three-electrode system was composed by an $\mathrm{Ag} / \mathrm{AgCl}$ reference electrode, a graphite lead as the auxiliary electrode, and a carbon nanotubes paste electrode as the working electrode. The following composition was used in the preparation of the working electrode: $55 \% \mathrm{~m} / \mathrm{m}$ of multiwall carbon nanotubes (Sigma-Aldrich) and $45 \% \mathrm{~m} / \mathrm{m}$ of mineral oil (Specsol). The electrode was assembled by compacting the carbon nanotubes paste at the tip of a $1 \mathrm{~mL}$ plastic syringe. The internal electric contact was made by a cleaned copper wire, which also acts as a plunger for paste extrusion. Renewal of the electrode surface was achieved by paste extrusion and smoothing onto a sulfite paper before each new measurement. All voltammograms were obtained in triplicate.

\section{Computational analysis}

All voltammetric data was processed using MATLAB ${ }^{\circledR}$ R2014 software ${ }^{33}$ with PLS Toolbox version 7.9.3. ${ }^{34}$ Raw voltammograms were pre-processed by cutting between -0.2 and $+0.9 \mathrm{~V}$, and applying Savitzky-Golay first derivative (window 15 points). Dataset comprising 80 voltammograms was divided into training $(n=52)$, validation $(n=14)$ and test $(\mathrm{n}=14)$ sets using the Kennard-Stone (KS) sample selection algorithm. ${ }^{35}$ The KS algorithm was applied separately to each class to extract a representative set of objects from a given class by maximizing the minimal Euclidean distance between the selected and the remaining objects. The training samples were used in the modeling procedure, whereas the test set was only used in the final evaluation of the classification. The optimum number of variables for SPA-LDA, SPA-QDA, GA-LDA, and GA-QDA was based on the average risk G of LDA/QDA misclassification. Such a cost function is calculated in the validation set as:

$\mathrm{G}=\frac{1}{\mathrm{~N}_{\mathrm{V}}} \sum_{\mathrm{n}=1}^{\mathrm{N}_{\mathrm{v}}} \mathrm{g}_{\mathrm{n}}$

where $\mathrm{N}_{\mathrm{V}}$ is the number of validation samples, and $\mathrm{g}_{\mathrm{n}}$ (risk of an incorrect classification of the object $x_{n}$ of the $n^{\text {th }}$ validation sample) is defined as

$g_{n}=\frac{r^{2}\left(x_{n}, m_{I(m)}\right)}{\min _{I(m) \neq I(n)} r^{2}\left(x_{n}, m_{I(m)}\right)}$

where $\mathrm{I}(\mathrm{n})$ is the index of the true class for the $\mathrm{n}^{\text {th }}$ validation object $\mathrm{x}_{\mathrm{n}}, \mathrm{m}_{\mathrm{I}(\mathrm{n})}$ the samples mean of their true class, $\mathrm{m}_{\mathrm{I}(\mathrm{m})}$ the samples mean of the closest wrong class, and $\mathrm{r}^{2}$ is the square of the Mahalanobis distance between the object $x_{n}$ and the mean of its class $\left(\mathrm{m}_{1(\mathrm{n})}\right)$.

Sensitivity (SENS, the confidence that a positive result for a sample of the label class is obtained), specificity (SPEC, the confidence that a negative result for a sample of non-label class is obtained), positive predictive value (PPV, measures the proportion of correctly assigned positive examples), negative predictive value (NPV, measures the proportion of correctly assigned negative examples), Youden's index (YOU, evaluates the classifier's ability to avoid failure), and the likelihood ratios $\operatorname{LR}(+)$ (the ratio between the probability to predict an example as positive when it is truly positive, 
and the probability to predict an example as positive when it is actually not positive) and LR(-) (the ratio between the probabilities to predict an example as negative when it is actually positive, and the probability to predict an example as negative when it is truly negative) were calculated as important quality parameters in test evaluation.

$$
\begin{aligned}
& \operatorname{SENS}(\%)=\left(\frac{\mathrm{TP}}{\mathrm{TP}+\mathrm{FN}}\right) \times 100 \\
& \operatorname{SPEC}(\%)=\left(\frac{\mathrm{TN}}{\mathrm{TN}+\mathrm{FP}}\right) \times 100 \\
& \mathrm{PPV}=\frac{\mathrm{TP}}{\mathrm{TP}+\mathrm{FP}} \\
& \mathrm{NPV}=\frac{\mathrm{TN}}{\mathrm{TN}+\mathrm{FN}} \\
& \mathrm{YOU}=\mathrm{SENS}-(1-\mathrm{SPEC}) \\
& \mathrm{LR}(+)=\frac{\mathrm{SENS}}{1-\mathrm{SPEC}} \\
& \mathrm{LR}(-)=\frac{1-\mathrm{SENS}}{\mathrm{SPEC}}
\end{aligned}
$$

$\mathrm{FN}$ is defined as false negative, $\mathrm{FP}$ as false positive, TP as true positive and $\mathrm{TN}$ as true negative.

\section{Fluorescence spectroscopy}

\section{Emission excitation matrix}

This procedure was performed for obtaining the individual EEM of control and contaminated with flunitrazepam classes. The sample spectra were acquired with an RF-5301 Shimadzu spectrofluorometer using a $0.5 \mathrm{~mm}$ quartz cuvette. The excitation and emission monochromator slit widths were fixed at 1.5 and $3 \mathrm{~nm}$, respectively. The samples were added to a cuvette with a $100 \mu \mathrm{L}$ micropipette. The cuvette was cleaned by ultra-pure water after each measurement. The temperature was kept at $25{ }^{\circ} \mathrm{C}$ throughout the experiments. For larval extract samples, the spectral surfaces of emission/excitation were obtained in the excitation range from 280 to $390 \mathrm{~nm}$ (10 $\mathrm{nm}$ steps) and in the emission range from 350 to $900 \mathrm{~nm}$ (1 $\mathrm{nm}$ steps). This protocol resulted in a data matrix size of $9 \times 322$ variables for each sample.

\section{Computational analysis}

All EEM data was processed using MATLAB® R2014 software $^{33}$ with PLS Toolbox version 7.9.3, ${ }^{34}$ EEMscat algorithm $^{36}$ and lab-made routines. The data was firstly pre-processed by removing Rayleigh and Raman scatterings using EEMscat algorithm and then mean centered before chemometric analysis.

The samples were divided into training $(n=20)$, validation $(\mathrm{n}=10)$ and test $(\mathrm{n}=10)$ sets using the KS sample selection algorithm. ${ }^{35}$ The training set was composed of 10 samples of class 1 (control) and 10 samples of class 2 (drugged with flunitrazepam); both validation and test sets had 5 samples of class 1 and 5 samples of class 2 . For classification, the data were initially unfolded and the first-order classification algorithms were tested. The unfolded procedure reshaped each EEM matrix with the size of $9 \times 322$ to a vector of size $1 \times 2898$. LDA, QDA, and SVM were applied to the data after principal component analysis (PCA) reduction. ${ }^{37} \mathrm{PCA}$ reduced the unfolded data to a few numbers of principal components (PCs), in which the scores on the selected PCs were used as input variables for classification.

In addition, second-order classification algorithms were applied to the original EEM matrices using PARAFAC-LDA and 2D-LDA algorithms. PARAFAC-LDA is based on a PARAFAC $^{38}$ decomposition of the three-way data of EEM matrices, followed by the application of LDA to the PARAFAC scores matrix. The PARAFAC decomposition is represented by: ${ }^{29}$

$\underline{\mathbf{X}}=\mathbf{A}(\mathbf{C}|\otimes| \mathbf{B})^{\mathrm{T}}+\underline{\mathbf{E}}$

in which $\underline{\mathbf{X}}$ represents the three-way EEM matrices; $\mathbf{A}$ is the PARAFAC scores; $\mathbf{B}$ is the PARAFAC loadings representing the excitation direction; $\mathbf{C}$ is the PARAFAC loadings representing the emission direction; $\underline{\mathbf{E}}$ is the residual tensor; and $\otimes$ represents the Khatri-Rao product.

The 2D-LDA algorithm is an adaptation of LDA to three-way data. The classification of a test sample is based on assigning its feature matrix $\mathbf{Y}_{\text {test }}$ to the class $\mathrm{p}^{*}$ with the smallest average distance: ${ }^{39}$

$\overline{\mathrm{d}}\left(\mathbf{Y}_{\text {test }}, \mathrm{C}_{\mathrm{p}^{*}}\right)=\min _{\mathrm{p}=1,2, \ldots, \mathrm{L}} \overline{\mathrm{d}}\left(\mathbf{Y}_{\text {test }}, \mathrm{C}_{\mathrm{p}}\right)$

in which $\overline{\mathrm{d}}\left(\mathbf{Y}_{\text {test }}, \mathrm{C}_{\mathrm{p}}\right)$ is the average distance between the test sample and the $\mathrm{N}_{\mathrm{p}}$ training samples of class $\mathrm{C}_{\mathrm{p}}$ can be calculated by:

$\overline{\mathrm{d}}\left(\mathbf{Y}_{\text {test }}, \mathrm{C}_{\mathrm{p}}\right)=\frac{1}{\mathrm{~N}_{\mathrm{p}}} \sum_{\mathrm{k} \in \mathrm{I}_{\mathrm{p}}} \mathrm{d}\left(\mathbf{Y}_{\text {test }}, \mathbf{Y}_{\mathrm{k}}\right)$

where $\mathrm{d}\left(\mathbf{Y}_{\text {test }}, \mathbf{Y}_{\mathrm{k}}\right)$ is the Euclidian distance between $\mathbf{Y}_{\text {test }}$ and the feature vector $\mathbf{Y}_{\mathrm{k}}$ of samples in the training set. The feature vector $\mathbf{Y}$ of each sample is calculated for a given data matrix $\mathbf{X}$ as: 
$\mathbf{Y}=\mathbf{X B}$

where $\mathbf{B}$ is a projection matrix obtained by maximizing Fisher's linear projection criterion; thus, maximizing the between-class over the within-class scatter matrices. ${ }^{39}$

\section{Statistical validation}

The statistical validation of the models built was evaluated according to the accuracy, sensitivity and specificity parameters. The accuracy represents the total number of samples correctly classified considering true and false negatives. This parameter is calculated as follows: ${ }^{40}$

$\operatorname{Accuracy}(\%)=\left(\frac{\mathrm{TP}+\mathrm{TN}}{\mathrm{TP}+\mathrm{FP}+\mathrm{TN}+\mathrm{FN}}\right) \times 100$

where TP stands for true positive; TN for true negative; $\mathrm{FP}$ for false positive; and $\mathrm{FN}$ for false negative.

\section{Results and Discussion}

\section{Electrochemical analysis}

In total, 80 voltammograms were acquired. The average differential pulse voltammograms for each class in the range of -1.0 to $+1.0 \mathrm{~V}$ presented similar shape (control, black line; flunitrazepam, red dashed line, Figure 1a). Baseline correction was performed in the voltammograms by using a B-spline interpolation function after baseline points established by first derivative Savitzky-Golay smoothing filter with a first-order polynomial (3-points windows size). Figure $1 \mathrm{~b}$ shows the baseline-corrected voltammograms.
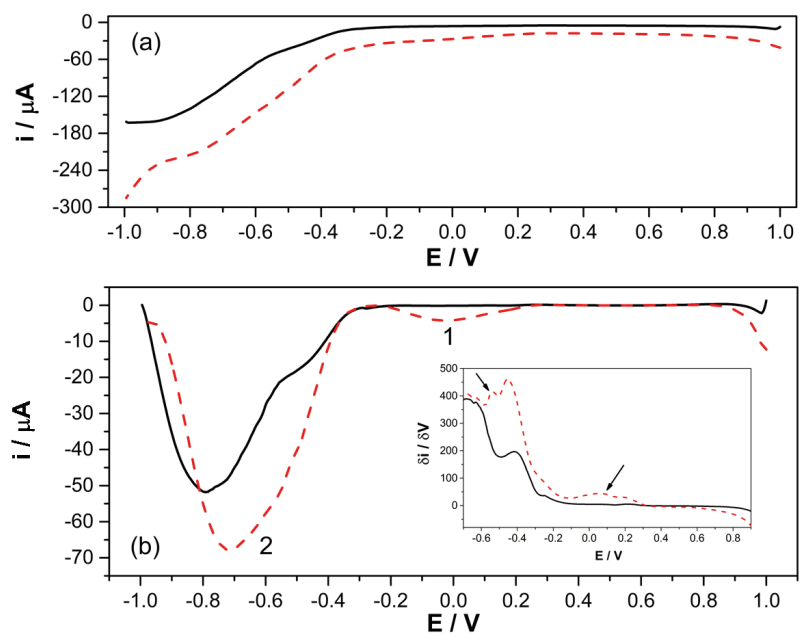

Figure 1. Average DPV data for control (black solid line) and flunitrazepam-contaminated (red dashed line) samples (a) before and (b) after baseline correction. Insert: voltammograms transformed by first-derivative. Arrows indicate the main differences in the mean voltammogram for each class.
The voltammogram related to flunitrazepam-contaminated samples shows two important differences compared to the voltammogram of the control samples. First, a broad less intense peak between -0.2 and $+0.2 \mathrm{~V}$, which could be attributed to a redox process of 7-aminoflunitrazepam, ${ }^{41}$ a pharmacologically active metabolite of flunitrazepam. The scan initiates at $+1.0 \mathrm{~V}$, promoting the oxidation of the amine group to hydroxylamine, which is back reduced to the amine, as described by equation 15 , and giving rise to peak 1 (Figure 1b).

$\mathrm{R}-\mathrm{NH}_{2}+\mathrm{H}_{2} \mathrm{O} \leftrightarrow \mathrm{R}-\mathrm{NHOH}+2 \mathrm{H}^{+}+2 \mathrm{e}^{-}$

The second difference is related to peak 2, composed of two redox processes partially overlapped in the voltammogram of control samples, related to the composition of sample matrix. In the average voltammogram of samples contaminated with flunitrazepam, peak 2 appears with higher current intensity and peak potential, which could be due to the contribution of the reduction of 7-nitro group of non-metabolized flunitrazepam to hydroxylamine ${ }^{32}$ according to equation 16 .

$\mathrm{R}-\mathrm{NO}_{2}+4 \mathrm{e}^{-}+4 \mathrm{H}^{+} \leftrightarrow \mathrm{R}-\mathrm{NHOH}+\mathrm{H}_{2} \mathrm{O}$

Insert of Figure $1 \mathrm{~b}$ shows the average voltammograms after Savitzky-Golay first derivative. One can see an additional peak between -0.3 and $-0.7 \mathrm{~V}$ in the voltammogram of contaminated samples, confirming a third redox process related to the presence of flunitrazepam. It is important to point out that the best results of classification were obtained with voltammograms transformed by first derivative and potential window from -0.2 to $+0.9 \mathrm{~V}$, i.e., by using information related only to peak 1 . Consequently, in postmortem specimens, the drug metabolized over time to 7-aminoflunitrazepam makes it a critical analyte for this study.

PCA was applied as an unsupervised exploratory method to visualize the distribution of the samples in the multidimensional space. Figure 2 presents the PCA scores plot. One can observe that approximately $98 \%$ of data variability was explained by two PCs, and there is a substantial dispersion and overlapping of the control and flunitrazepam-contaminated samples. Following, the application of supervised algorithms of classification, more specifically LDA or QDA, was performed to build multivariate classification models. These were adopted systematically to discriminate control vs. contaminated samples based on DPV.

Table 1 presents the figures of merit of the obtained classification models for each class. According to the 


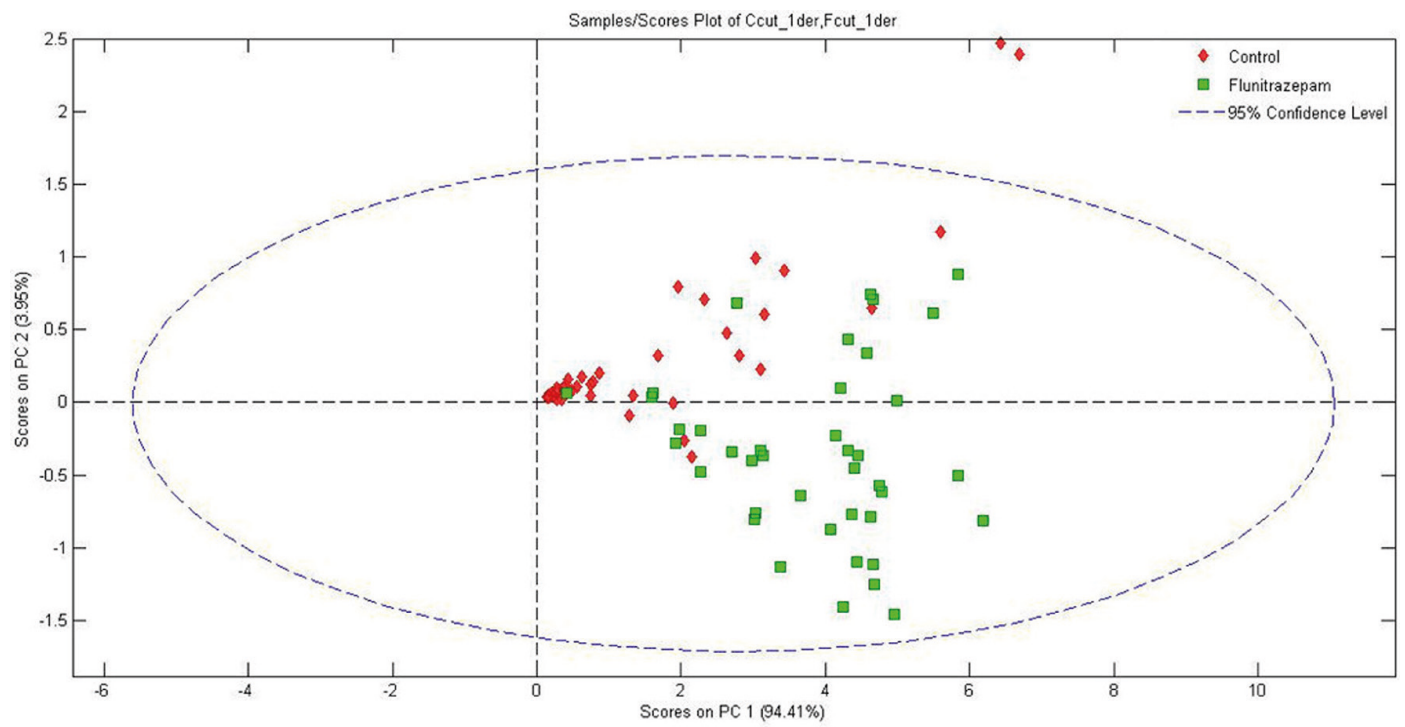

Figure 2. Exploratory analysis with PCA: PC1 $\times$ PC2 scores plot.

results shown in Table 1 for control samples, it is possible to conclude that this class presents a more homogeneous composition measured by DPV, always reaching ideal scores by any of the evaluated methods. On the other hand, for flunitrazepam-contaminated class, the figures of merit obtained with QDA models were better than those obtained with the LDA ones. This could be related to the greater heterogeneity of such class due to the more complex process of assimilation and metabolization of flunitrazepam by larvae, which required a quadratic discriminant function to perform a better classification. For example, sensitivity values of QDA models were superior to that obtained with LDA, being 92.9, 92.9, and 100\% for PCA-QDA, SPA-QDA, and GA-QDA, respectively. Considering all figures of merit of Table 1, GA-QDA model presented the maximum efficiency for classification.

Table 1. Figures of merit of linear (LDA) and quadratic discriminant analysis (QDA) classification models for each class

\begin{tabular}{|c|c|c|c|c|c|c|c|c|c|}
\hline \multirow[t]{2}{*}{ Algorithm } & \multicolumn{2}{|c|}{ Control } & \multicolumn{2}{|c|}{ Flunitrazepam } & \multirow[t]{2}{*}{ Algorithm } & \multicolumn{2}{|c|}{ Control } & \multicolumn{2}{|c|}{ Flunitrazepam } \\
\hline & SENS & 100 & SENS & 78.6 & & SENS & 100 & SENS & 92.9 \\
\hline & SPEC & 100 & SPEC & 78.6 & & SPEC & 100 & SPEC & 100 \\
\hline & PPV & 100 & PPV & 78.6 & & PPV & 100 & PPV & 100 \\
\hline \multirow[t]{7}{*}{ PCA-LDA } & NPV & 100 & NPV & 78.6 & PCA-QDA & NPV & 100 & NPV & 93.3 \\
\hline & YOU & 100 & YOU & 57.1 & & YOU & 100 & YOU & 92.9 \\
\hline & $\mathrm{LR}(+)$ & 0.0 & $\mathrm{LR}(+)$ & 0.0 & & $\mathrm{LR}(+)$ & 0.0 & $\mathrm{LR}(+)$ & 0.0 \\
\hline & $\mathrm{LR}(-)$ & 0.0 & $\mathrm{LR}(-)$ & 0.3 & & $\mathrm{LR}(-)$ & 0.0 & $\mathrm{LR}(-)$ & 0.1 \\
\hline & SENS & 100 & SENS & 85.7 & & SENS & 100 & SENS & 92.9 \\
\hline & SPEC & 100 & SPEC & 85.7 & & SPEC & 100 & SPEC & 100 \\
\hline & PPV & 100 & PPV & 85.7 & & PPV & 100 & PPV & 100 \\
\hline \multirow[t]{7}{*}{ SPA-LDA } & NPV & 100 & NPV & 85.7 & SPA-QDA & NPV & 100 & NPV & 93.3 \\
\hline & YOU & 100 & YOU & 71.4 & & YOU & 100 & YOU & 92.9 \\
\hline & $\mathrm{LR}(+)$ & 0.0 & $\operatorname{LR}(+)$ & 0.0 & & $\mathrm{LR}(+)$ & 0.0 & $\mathrm{LR}(+)$ & 0.0 \\
\hline & $\mathrm{LR}(-)$ & 0.0 & $\operatorname{LR}(-)$ & 0.2 & & $\mathrm{LR}(-)$ & 0.0 & $\mathrm{LR}(-)$ & 0.1 \\
\hline & SENS & 100 & SENS & 78.6 & & SENS & 100 & SENS & 100 \\
\hline & SPEC & 100 & SPEC & 100 & & SPEC & 100 & SPEC & 100 \\
\hline & PPV & 100 & PPV & 100 & & PPV & 100 & PPV & 100 \\
\hline \multirow[t]{4}{*}{ GA-LDA } & NPV & 100 & NPV & 82.4 & GA-QDA & NPV & 100 & NPV & 100 \\
\hline & YOU & 100 & YOU & 78.6 & & YOU & 100 & YOU & 100 \\
\hline & $\mathrm{LR}(+)$ & 0.0 & $\operatorname{LR}(+)$ & 0.0 & & $\operatorname{LR}(+)$ & 0.0 & $\operatorname{LR}(+)$ & 0.0 \\
\hline & $\mathrm{LR}(-)$ & 0.0 & $\operatorname{LR}(-)$ & 0.2 & & $\mathrm{LR}(-)$ & 0.0 & $\operatorname{LR}(-)$ & 0.0 \\
\hline
\end{tabular}

SENS: sensitivity; SPEC specificity; PPV: positive predictive value; NPV: negative predictive value; YOU: Youden's index; LR(+): positive likelihood ratio; $L R(-)$ : negative likelihood ratio. 




Figure 3. The discriminant function calculated by GA-QDA model for control (purple) versus flunitrazepam-contaminated (black) samples.

The excellent discrimination power obtained for GA-QDA model is presented in Figure 3. GA-QDA model employed 13 variables (Figure 4). Four selected potentials seem to be of particular interest. Variables $-0.02,+0.14,+0.28$, and $+0.30 \mathrm{~V}$ are located at peak 1 , and model the influence of the presence of the metabolite 7-aminoflunitrazepam in differentiating each class with success. The other nine variables are located at the baseline and model the change on background current regarding the presence of flunitrazepam and its metabolite.

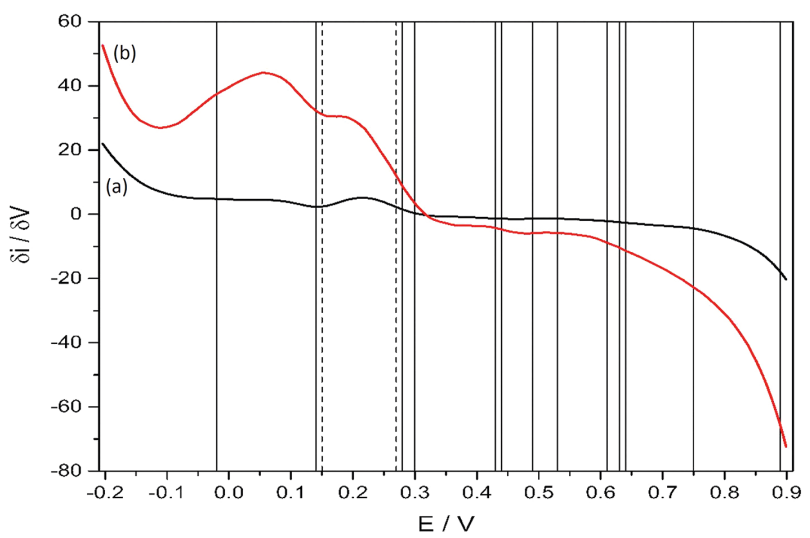

Figure 4. Average first-derivative voltammograms of each class ((a, black) control; (b, red) flunitrazepam) and the variables selected by GA-QDA (solid vertical lines) and SPA-QDA (dashed vertical lines).

SPA-QDA model employed two variables (Figure 4). Despite the lower classification efficiency offered by the SPA-QDA model, it can be considered that its results were satisfactory given the lower number of variables selected, which implies greater robustness when compared to GA-QDA. Examination of the selected potentials showed that the model used information associated to the presence of the drug detected by DPV in the contaminated samples.

\section{Fluorescence analysis}

Average EEM data for class 1 (control) and class 2 (larvae with flunitrazepam) are shown in Figure 5. As can be seen, a visual inspection cannot reveal significant differences between the two classes, since both profiles seem very similar.

A closer look into the excitation and emission profiles for each EEM can be seen in Figure 6. As demonstrated by Figure 6, both excitation and emission profiles for control and flunitrazepam contaminated samples are much superposed among the samples, where no clear separation can be observed.

For an initial assessment, each EEM data matrix was unfolded into a vector and first-order classification algorithms were tested. LDA, QDA and SVM were applied to the PCA scores on the first 10 PCs $(99.36 \%$ of cumulative variance). The results are shown in Table 2.

Table 2 shows that first-order classification algorithms did not successfully discriminate the two classes. The best accuracy and sensitivity was found with LDA $(60 \%)$ and the best specificity with QDA (80\%). However, overall these values are unsatisfactory for entomotoxicology applications, since the gold method using HPLC reaches values above $90 \%$ sensitivity and specificity. New methodologies such as 2D-LDA are strongly able to achieve the efficiency of gold methodologies and have a lower cost, require less sample preparation and analysis time, and keep the analytical accuracy at acceptable levels for expert requirements. 

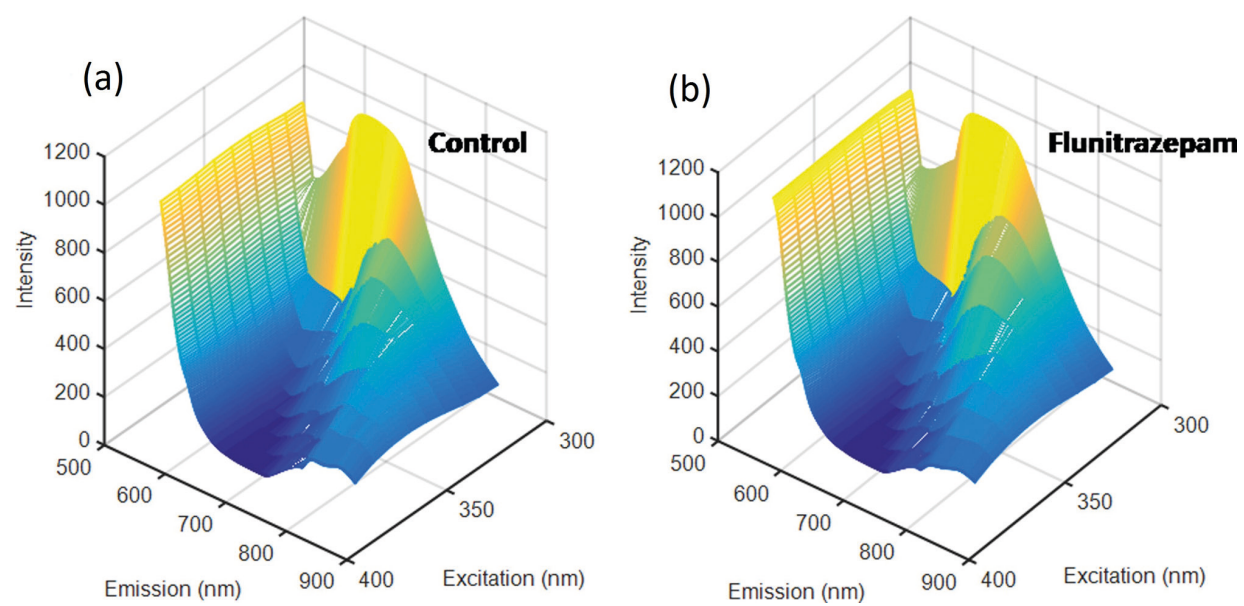

Figure 5. Average EEM data for (a) control and (b) flunitrazepam-contaminated samples.
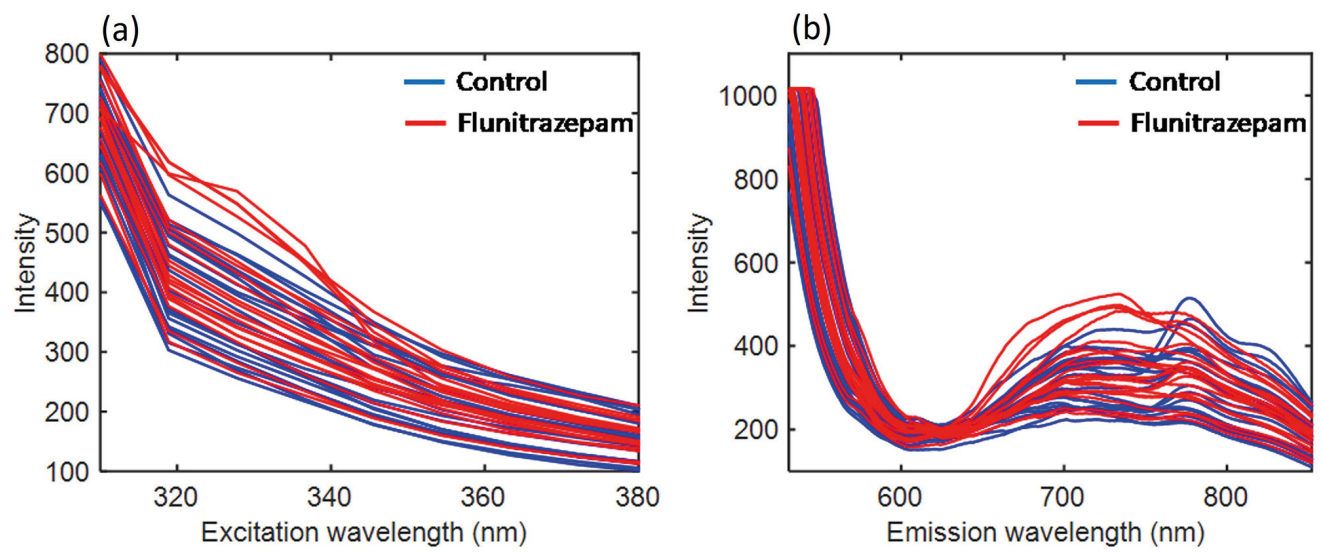

Figure 6. (a) Excitation and (b) emission profiles for control (blue) and flunitrazepam-contaminated (red) samples.

Table 2. Figures of merit calculated using different classification methods applied to the principal component analysis (PCA) scores of unfolded fluorescence excitation-emission matrix (EEM) data

\begin{tabular}{lccc}
\hline Classifier & Accuracy / $\%$ & Sensitivity / $\%$ & Specificity / $\%$ \\
\hline LDA & 60.0 & 60.0 & 60.0 \\
QDA & 50.0 & 20.0 & 80.0 \\
SVM & 50.0 & 40.0 & 60.0 \\
\hline
\end{tabular}

LDA: linear discriminant analysis; QDA: quadratic discriminant analysis; SVM: support vector machines.

Second-order classification algorithms were applied by means of PARAFAC-LDA and 2D-LDA. PARAFAC-LDA was built with 5 components, and 2D-LDA was built with 2 factors. Their classification results are shown in Table 3.

The overall classification performance of PARAFAC-LDA was very similar to that obtained using first-order algorithms (Table 2), in which poor discrimination between control and flunitrazepam-contaminated samples were observed. On the other hand, 2D-LDA presented a perfect performance with classification rates equal to $100 \%$ for all figures of merit. The 2D-LDA discriminant function
Table 3. Figures of merit calculated using PARAFAC-LDA and 2D-LDA applied to the three-way fluorescence excitation-emission matrix (EEM) data

\begin{tabular}{lccc}
\hline Classifier & Accuracy $/ \%$ & Sensitivity / $\%$ & Specificity / \% \\
\hline PARAFAC-LDA & 50.0 & 40.0 & 60.0 \\
2D-LDA & 100 & 100 & 100 \\
\hline
\end{tabular}

PARAFAC: parallel factor analysis; LDA: linear discriminant analysis.

(DF) plot is shown in Figure 7, where a perfect separation of the two classes is clearly observed.

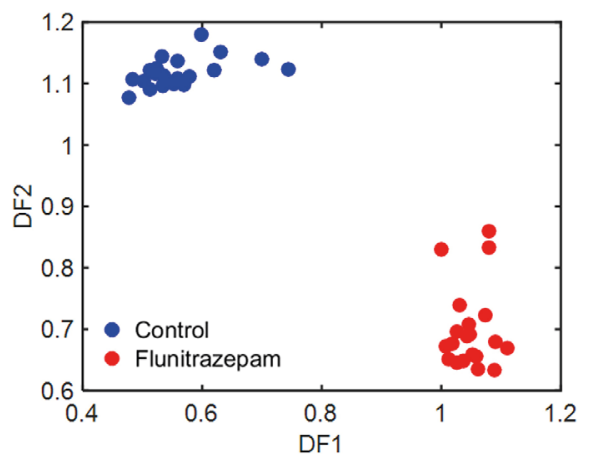

Figure 7. Discriminant function (DF) plot for factor 1 versus factor 2. 
2D-LDA has been reported as being superior to other classification methods such as PARAFAC in many chemical applications. ${ }^{39}$ Second-order classification algorithms seem superior to their unfolded versions due to the loss of spatial distribution information during unfolding procedure..$^{40}$ Therefore, the use of two-dimensional classification algorithms is the best option to analyze EEM data.

\section{Conclusions}

The results of this study show that differential pulse voltammetry combined with multivariate classification may be an alternative tool for the detection of flunitrazepam in necrophagous larvae. We report a fast, clean and low-cost method, which involves minimum samples preparation to classify the specimens. In this report, the resulting GA-QDA model successfully detected flunitrazepam providing $100 \%$ accuracy and specificity. This method was completely validated, showing potential for use as an official methodology for entomological methods. EEM fluorescence spectroscopy with 2D-LDA was effective to differentiate control and larval extract drugged with flunitrazepam for an entomotoxicology application. Using this methodology, $100 \%$ accuracy, sensitivity and specificity were found for discriminating the two classes. It represents a fast, precise and low-cost method for forensic analysis of larval extract, having a minimum sample preparation and with a small amount of sample required for analysis. This study is restricted to a local level application due to the number of samples used in the experiment and the environmental conditions submitted to the insects. Future studies are needed for the purpose of increasing sample space aiming at a global model, and may enable the analysis of new drugs for forensic interest.

\section{Acknowledgments}

Leomir A. S. de Lima would like to acknowledge the financial support from PPGQ/UFRN/CAPES for his fellowship. Camilo L. M. Morais would like to thank CAPES, Brazil (Doutorado Pleno no Exterior, grant 88881.128982/2016-01) for financial support. Kássio M. G. Lima would like to acknowledge the CNPq grant (305962/2014-0) for financial support. Sherlan G. Lemos would like to acknowledge the CNPq grant (301662/2015-0) for financial support.

\section{References}

1. Amendt, J.; Campobasso, C. P.; Gaudry, E.; Reiter, C.; LeBlanc, H. N.; Hall, M. J. R.; Int. J. Leg. Med. 2007, 121, 90.
2. Catts, E. P.; J. Agric. Entomol. 1992, 9, 245.

3. Lord, W. D.; Rodriquez, W. C.; Prosecutor 1989, 22, 41.

4. Benecke, M.; Forensic Sci. Int. 2001, 120, 2.

5. de Santana, C. S.; Siquieroli, D.; Boas, V.; Ceciliana 2012, 4, 31.

6. Gosselin, M.; Wille, S. M. R.; Fernandez, M. M. R.; Di Fazio, V.; Samyn, N.; De Boeck, G.; Bourel, B.; Forensic Sci. Int. 2011, 208, 1 .

7. Wallace, D. R.; Toxicol. Forensic Med. Open J. 2017, SE1. Available at https://www.openventio.org/wp-content/uploads/ Evolution-of-Forensic-Entomotoxicology-TFMOJ-SE-1-e001. pdf, accessed in July 2018.

8. Introna, F.; Campobasso, C. P.; Goff, M. L.; Forensic Sci. Int. 2001, 120, 42.

9. Goff, M.; Lord, W.; Am. J. Forensic Med. Pathol. 1994, 15, 51.

10. Smith, M. L.; Vorce, S. P.; Holler, J. M.; Shimomura, E.; Magluilo, J.; Jacobs, A. J.; Huestis, M. A.; J. Anal. Toxicol. 2007, 31, 237.

11. Oliveira, J. S.; Baia, T. C.; Gama, R. A.; Lima, K. M. G.; Microchem. J. 2014, 115, 39.

12. de Lima, L. A. S.; Baia, T. C.; Gama, R. A.; Lima, K. M. G.; NIR News 2014, 25, 5. DOI: 10.1255/nirn.1489.

13. Huitle, C. A. M.; Fernandes, N. S.; Lopes, M. C.; Quiroz, M. A.; Port. Electrochim. Acta 2010, 28, 39.

14. Bagalkoti, J. T.; Pattar, V. P.; Nandibewoor, S. T.; J. Electrochem. Sci. Eng. 2017, 7, 77.

15. Batchelor-McAuley, C.; Katelhon, E.; Barnes, E. O.; Compton, R. G.; Laborda, E.; Molina, A.; ChemistryOpen 2015, 4, 224.

16. Branica, G.; Metiko, M.; Omanovi, D.; Croat. Chem. Acta 2006, $79,77$.

17. Cuculi, V.; Pizeta, I.; Branica, M.; J. Electroanal. Chem. 2005, $583,140$.

18. Farghaly, O. A. E.; J. Pharm. Biomed. Anal. 2000, 23, 783.

19. Farghaly, O. A.; Hameed, R. S. A.; Abu-Nawwas, A. H.; Int. J. Electroanal. Chem. 2014, 25, 37.

20. Frenke, J. P.; De Zeeuw, R. A.; J. Anal. Toxicol. 1976, 1, 291.

21. Hoang, V. D.; Huyen, D. T.; Phuc, P. H.; J. Anal. Methods Chem. 2013, 2013, article ID 367914. DOI: 10.1155/2013/367914.

22. Yardim, Y.; Levent, A.; Zühre, S.; Talanta 2011, 85, 441.

23. Zayed, S. I. M.; Issa, Y. M.; Bioelectrochemistry 2009, 75, 9.

24. Christensen, J. H.; Hansen, A. B.; Mortensen, J.; Andersen, O.; Anal. Chem. 2005, 77, 2210.

25. Orzel, J.; Daszykowski, M.; Grabowski, I.; Zaleszczyk, G.; Sznajder, M.; Fuel 2014, 117, 224.

26. Xu, B.; Ye, Y.; Liao, L.; Forensic Sci. Res. 2017, DOI: 10.1080/20961790.2017.1349600.

27. Wu, W.; Mallet, Y.; Walcazak, B.; Penninckx, W.; Massart, D. L.; Heuerding, S.; Erni, F.; Anal. Chim. Acta 1996, 329, 257.

28. Cortes, C.; Vapnik, V.; Mach. Learn. 1995, 20, 273.

29. Costa, F. S. L.; Silva, P. P.; Morais, C. L. M.; Theodoro, R. C.; Arantes, T. D.; Lima, K. M. G.; Anal. Methods 2017, 9, 3968. 
30. Li, M.; Yuan, B.; Pattern Recognit. Lett. 2005, 26, 527.

31. Tirapelli, C. R.; Fukada, S. Y.; Yogi, A.; Chignalia, A. Z.; Tostes, R. C.; Bonaventura, D.; Lanchote, V. L.; Cunha, Q.; de Oliveira, A. M.; Braz. J. Pharm. 2008, 153, 468.

32. Shinomiya, K.; Shigemoto, Y.; Omichi, J.; Utsu, Y.; Mio, M.; Kamei, C.; Psychopharmacology 2004, 173, 203.

33. $M A T L A B^{\circledast} R 2014$, version R2014a; Mathworks, Natrick, USA, 2014.

34. PLS Toolbox, version 7.9.3; Eigenvector Research, Inc., Manson, USA, 2014.

35. Kennard, R. W.; Stone, L. A.; Technometrics 1969, 11, 137.

36. Bahram, M.; Bro, R.; Stedmon, C.; Afkhami, A.; J. Chemom. 2006, 20, 99.
37. Bro, R.; Smilde, A. K.; Anal. Methods 2014, 6, 2812.

38. Bro, R.; Chemom. Intell. Lab. Syst. 1997, 38, 149.

39. da Silva, A. C.; Soares, S. F. C.; Insausti, M.; Galvão, R. K. H.; Band, B. S. F.; de Araújo, M. C. U.; Anal. Chim. Acta 2016, 938, 53.

40. Morais, C. L. M.; Lima, K. M. G.; Chemom. Intell. Lab. Syst. 2017, $170,1$.

41. Garcia-Gutierrez, E.; Lledo-Fernandez, C.; Chemosensors 2013, 1,68 .

Submitted: February 26, 2018

Published online: July 18, 2018 